\title{
Right Atrial Thrombus Associated with Central Venous Catheter after Surgical Repair of Co-arctation of Aorta
}

\author{
Smriti Mahaju Bajracharya ${ }^{1}$, Sandeep Sapkota ${ }^{1}$ \\ 'Department of Cardiac Anaesthesia, Shahid Gangalal National Heart Centre, Bansbari, Kathmandu, Nepal.
}

Corresponding Author: Smriti Mahaju Bajracharya

Department of Cardiac Anaesthesia, Shahid Gangalal National Heart Centre, Bansbari, Kathmandu, Nepal.

Email: smriti.mahaju@gmail.com

ORCID ID NO: 0000-0002-9118-2633

Cite this article as: Bajracharya S. M., Sapkota S. Right Atrial Thrombus Associated with Central Venous Catheter after Surgical Repair of Co-arctation of Aorta. Nepalese Heart Journal 2021; Vol 18(2), 69-71.

Submission date: $28^{\text {th }}$ August, 2021

Accepted date: $3^{\text {th }}$ October, 2021

\begin{abstract}
Central venous catheterization (CVC) is a routine and essential procedure performed in patients undergoing cardiac surgery. However, its use is not without complications ranging from mechanical to infectious to thrombotic in nature. Catheter-related right atrial thrombosis (CRAT) is usually underreported as it is commonly small in size and asymptomatic. Nevertheless, CRAT might lead to a potentially severe and life threatening complication in both adult and young patients. Up to $30 \%$ of patients with CVCs are estimated to be affected by CRAT. Anticoagulation and subsequently catheter removal were recommended as a first-line therapy and have suggested that no intervention may be associated with a high mortality rate.

Here, we report a case of a 3-months-old male child who underwent coarctation of aorta repair and was diagnosed with right atrial thrombosis associated with CVC in postoperative period and anticoagulated with unfractionated heparin. The thrombus was followed up closely and when there was no further decrease in the size of the mass around 9 days after the diagnosis, the CVC was removed. The heparin was continued even after removal of CVC for further 7 days and patient was eventually discharged from the hospital.
\end{abstract}

Keywords: Central venous catheterization, Catheter-related right atrial thrombosis, Coarctation of aorta

DOI: https://doi.org/10.3126/njh.v18i2.40409

\section{Introduction}

Central venous catheterization $(\mathrm{CVC})$ is a routine and essential procedure performed in patients undergoing cardiac surgery, which allows measurement of hemodynamic variables, delivery of medications, nutritional support and fluids during peri-operative period. The right internal jugular vein (IJV) is the preferred site of cannulation, however, its use is not without complications. CVC may have complications ranging from mechanical to infectious to thrombotic in nature. Catheter-related right atrial thrombosis (CRAT) is usually underreported as it is commonly small in size and asymptomatic. Nevertheless, CRAT might lead to a potentially severe and life threatening complication in both adult and young patients $^{1}$. Up to $30 \%$ of patients with CVCs are estimated to be affected by CRAT1. As there are no uniform and standard guidelines regarding the management of CRAT, there exists a wide variation in clinical practice. Anticoagulation and subsequently catheter removal were recommended as a first-line therapy and have suggested that no intervention may be associated with a high mortality rate ${ }^{2,3}$. Trans-esophageal and trans-thoracic echocardiography (TTE) are mainly used for CRAT diagnosis ${ }^{3}$. There are limited evidence-based guidelines for the best diagnostic approach and preventive measures for CRT as early recognition and treatment may have possible survival benefit.

Here, we report a case of a 3-months-old male child who underwent coarctation of aorta repair and was diagnosed with right atrial thrombosis associated with CVC in postoperative period and anticoagulated with unfractionated heparin. The thrombus was followed up closely and when there was no further decrease in the size of the mass around 9 days after the diagnosis, the $\mathrm{CVC}$ was 
removed. The heparin was continued even after removal of CVC for further 7 days and patient was eventually discharged from the hospital.

\section{Case Presentation}

A 3-months-old male child was diagnosed incidentally with coarctation of aorta during investigations performed when the child was admitted for aspiration with shortness of breath. On Chest X-ray, cardiomegaly was noticed. Hence, TTE was done which revealed coarctation of aorta. The patient then was scheduled electively for resection and end-to-end anastomosis of the coarctation of aorta. Preoperative vitals were within normal limits and routine laboratory tests revealed hemoglobin of $9.9 \mathrm{gm} \%$ with platelet counts of $333000 / \mathrm{cc}$. In the operating room, standard monitors were applied. As per institutional protocol general anaesthesia was induced uneventfully. A Triple Lumen CVC (5 French and $7 \mathrm{~cm}$ ) was inserted via the right IJV without any difficulty before operation. The operation underwent uneventfully and the patient was transferred to the pediatric intensive care unit with dopamine and nitroglycerin infusion.

Around 4 hours after the operation, the patient was taken for reexploration as haemorrhagic pericardial effusion was seen on echo screening which couldn't be explained as end-to-end anastomosis of the coarctation of aorta is extra-pericardial procedure. CVC was in correct position and the source of the bleeding could not be elucidated. Patient was extubated 8 hours after the initial operation fulfilling extubation criteria. On postoperative day (POD) 1 , hemoglobin was on the lower side $(7.3 \mathrm{gm} \%)$ and blood transfusion was started. Around after 3 hours into the blood transfusion ( $26 \mathrm{hrs}$ after the surgery), patient developed rashes all over the body and temp of 101 degrees F. Blood transfusion was stopped immediately. The most possible cause maybe is the transfusion-associated hyperkalemic cardiac arrest with rapid RBC administration. Most published cases of transfusion-associated hyperkalemic cardiac arrest have occurred in pediatric patients ${ }^{4-6}$. Arterial blood gas analysis revealed a potassium of $7.33 \mathrm{mEq} / \mathrm{L}$ most possibly due to blood transfusion and immediately treatment for hyperkalemia with Lasix, calcium, dextrose, and insulin was initiated. After 1 hour, potassium was consistently high of $7.38 \mathrm{mEq} / \mathrm{L}$. During this time, patient was also noted to have focal seizure (unrolling of eyes) for 30 secs which was treated with midazolam $0.5 \mathrm{mg}$ which may be secondary to hypoxia. Patient had a cardiac arrest and cardiopulmonary resuscitation (CPR, ADR 10 mcg*8 ) was performed for about 20 mins; the trachea was intubated and the patient was revived. Potassium levels decreased to $6.44 \mathrm{mEq} / \mathrm{L}$ immediately after CPR. Patient was started on dopamine and adrenaline infusions. After 2 hours, potassium level further decreased to $4.05 \mathrm{mEq} / \mathrm{L}$ and the patient became hemodynamic stable. On POD 2, patient again had several episodes of seizures and phenytoin and levera were started. On POD 3, all the inotropes were gradually tapered off.

Routine TTE examination on POD 3 revealed a small round mass in the right atrium. The mass was confined around the CVC tip measuring $6.5 \mathrm{~mm} * 3.4 \mathrm{~mm}$. A diagnosis of thrombus was made and the patient was started on anticoagulation with unfractionated heparin. TTE was performed every day to assess the thrombus. Since there was an ongoing need for central assess and the catheter was non-infected and well-functioning, decision to continue with same CVC line was made. The plan was to continue with medical management with unfractionated heparin. On POD 5, 3rd day of starting unfractionated heparin, the thrombus around CVP tip decreased and measured $5.8 \mathrm{~mm} * 3.8 \mathrm{~mm}$. On 7th POD, the thrombus measured $5.4 \mathrm{~mm} * 3.1 \mathrm{~mm}$. On 6th POD, CT scan of head revealed diffuse cerebral and cerebellum edema. The patient was extubated on 7th POD. The thrombus remained the same size for the next 3 days. On POD 11, CVC was removed taking precautions. The patient was monitored for the signs of any thromboembolic consequences. There was no sessile or remaining free thrombus on right atrium after taking out the catheter on TTE. There were no signs of thromboembolic events till 48 hours of removing CVC and patient was shifted out of ICU to ward. His six days stay at ward was uneventful and was discharged from the hospital. The heparin was stopped 1 day before discharge.

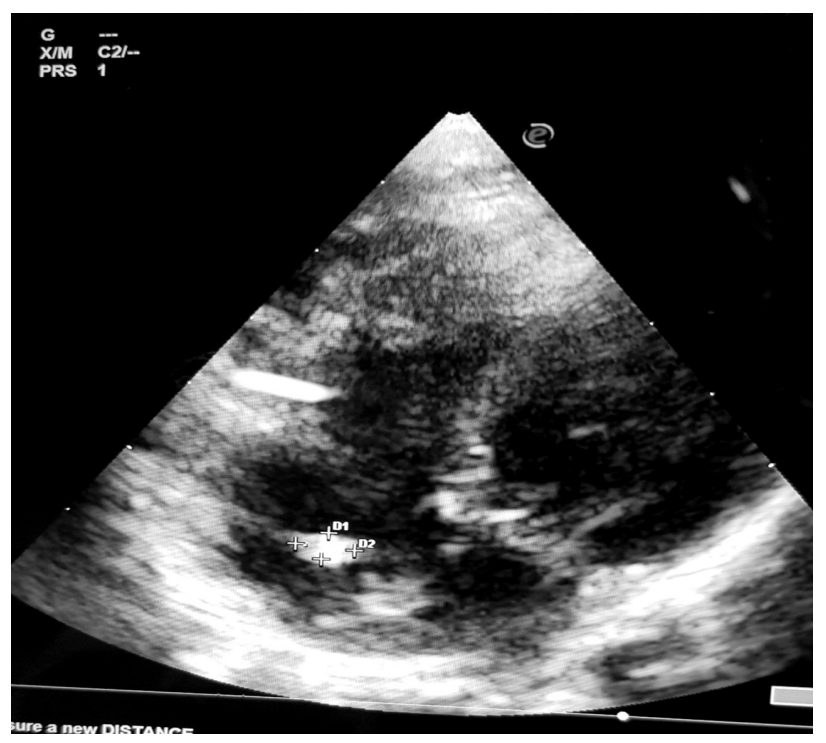

Figure 1: TTE showing mass around the CVP tip in the right atrium

\section{Discussion}

$\mathrm{CVC}$ is routinely used in all adult and paediatric cardiac surgical procedures. CRAT may be relatively common than actually reported in literature, as almost two third of the cases are asymptomatic and hence under-reported. Right atrial (RA) thrombus may be complicated by cardiogenic shock, pulmonary embolism or cardiac arrest, which may result in a high mortality rate ranging from $28 \%$ to $31 \% \%^{7-9}$.CRAT has a high association of the catheter tip position in the RA as in our case $\mathrm{c}^{8,10,11}$. In a study by Gilon et al ,they found that the formation of thrombi was highly associated with the catheter tip position in the RA, in contrast to their positioning in the superior vena cava or in its junction with the RA ${ }^{11}$. The catheter tip in the RA can provoke a mechanical irritation of the atrial wall due to repeatedly brushing against its wall. The resulting endothelial injury may result in activation of the coagulation cascade and thrombus formation ${ }^{7,12}$. The severity of RA thrombus is much less in paediatric patients, leading to less hemodynamic compromise and pulmonary embolism ${ }^{10}$. Two types of RA thrombus, type A and $\mathrm{B}$ have been described in the literature. Type A which is mobile, is thromboembolic in nature and has a higher risk of pulmonary embolism as compared to type $\mathrm{B}^{2}$. Type $\mathrm{B}$ thrombus on the other hand forms around foreign bodies like CVC in the $\mathrm{RA}^{2}$. RA thrombus type B may be asymptomatic, as in our case. As already discussed, majority of the cases are undiagnosed due to the absence of symptoms. They are diagnosed either accidently when looking for other pathologies or if there is associated symptoms arising from complications of RA thrombus, which include pulmonary embolism, systemic embolism, infected thrombi, and hemodynamic compromise ${ }^{2}$. These complications may result in a higher mortality in type B RA thrombus.

Until now, the treatment strategies for CRAT have a limited evidence with only a handful of case reports and series. The treatment of CRAT in most cases is systemic anticoagulation with 
or without line removal, however, due to a lack of good quality data, its duration is again controversial. Study by Stavroulopoulos et al ${ }^{2}$. reported for several possible approaches to the management options for CRAT, which include Oral/systemic anticoagulation, surgical thrombectomy (open heart, percutaneous), or thrombolysis ${ }^{2}$ followed by the removal of CVP catheter. Removal of the device should be decided by clinical necessity for venous access or by evidence of pulmonary embolism. In our case, we proceeded with the removal of catheter when there was no further reduction in the size of mass for several days. Anticoagulation was continued until the hospital day considering the fact that our patient was not a high risk and the $\mathrm{CV}$ catheter was removed. As no treatment modalities have been shown superior to one another with one having better outcome than another, we did not continue anticoagulation after the hospital discharge and since the catheter was removed; the recurrence may be low without other ongoing risk factor. Korones et al believed that a small sized thrombus did not need any intervention, even though no regression was seen after 1 year, and that a moderatesized thrombus only needed medication, such as warfarin, instead of surgical thrombectomy ${ }^{10}$. In a Letter to the Editor from Kroger et al, 42 patients $(80 \%)$ with CVT received only heparin intravenously, with effective prolongation of the partial thromboplastin time, or LMWH subcutaneously ${ }^{13}$. Studies have shown that surgical thrombectomy seems to be slightly superior to anticoagulation, but it is indicated only in case of contraindication or inefficacy of the anticoagulation, thrombus size $\geq 6 \mathrm{~cm}$, cardiac abnormalities or endocarditis. Several factors related to the host (underlying cardiac arrest), instrumentation (CVC) and treatment (heparin exposure) may have acted synergistically and resulted in thrombus formation. Hence, the formation of clot may be a multifactorial event.

We would like to emphasize that management strategies should be individualized based on the patient's risk factor for thrombosis, size of the thrombus, complications arising from it. In our case the patient was discharged from the hospital without residual sequel although we opted for more conservative treatment. In addition, decision to remove or not to remove the catheter is not without complications. The literature may underestimate the incidence of thromboembolic complications related to $\mathrm{CVC}$ because the diagnosis may not be considered in symptomatic and, especially, in asymptomatic patients, or may be missed by TTE.

In conclusion, CRAT is an underdiagnosed and life-threatening complication of CVCs and may be associated with a high mortality from its complications; this may not be as rare as we previously thought, if the catheter tip is located in the RA. In pediatric patients who have small to moderately sized thrombi, medical management is preferred. Nonetheless, those with large sized thrombi whether be adults or children, surgical thrombectomy is considered safe and effective treatment ${ }^{14}$. This present case highlights the important role of TTE and anticoagulation in the diagnosis and management respectively of CRAT.

\section{Acknowledgements}

Dr Deepak Tempe and Dept of cardiac anesthesia and cardiac surgery, Shahid Gangalal National Heart Centre.

\section{References:}

1. Baskin JL, Pui CH, Reiss U, et al. Management of occlusion and thrombosis associated with long-term indwelling central venous catheters. Lancet 2009; 374: 159-169.

2. Stavroulopoulos A, Aresti V and Zounis C. Right atrial thrombi complicating haemodialysis catheters. A metaanalysis of reported cases and a proposal of a management algorithm. Nephrol Dial Transplant 2012; 27(7): 2936-2944.

3. Negulescu O, Coco M, Croll J, et al. Large atrial thrombus formation associated with tunneled cuffed hemodialysis catheters. Clin Nephrol 2003; 59(1): 40-46.

4. Chen CH, Hong CL, Kau YC, Lee HL, Chen CK, Shyr MH. Fatal hyperkalemia during rapid and massive blood transfusion in a child undergoing hip surgery-a case report. Acta Anaesthesiol Sin 1999;37:163-6

5. Buntain SG, Pabari M. Massive transfusion and hyperkalaemic cardiac arrest in craniofacial surgery in a child. Anaesth Intensive Care 1999;27:530-3

6. Ivens D, Camu F. Sudden hyperkalemia during cardiopulmonary bypass with hypothermic cardiac arrest in an infant. J Cardiothorac Vasc Anesth 1996;10:258-60

7. Alhaddad IA, Soubani AO, Brown EJ Jr, Jonas EA, Freeman I. Cardiogenic shock due to huge right atrial thrombus. Chest 1993;104:1609-10.

8. Kingdon EJ, Holt SG, Davar J, et al. Atrial thrombus and central venous dialysis catheters. Am J Kidney Dis 2001; 38:631-9.

9. Forauer AR, Bocchini TP, Lucas ED, Parker KR. Giant right atrial thrombus: a life-threatening complication of longterm central venous access catheters. J Vasc Interv Radiol 1998;9:519-20.

10. Korones DN, Buzzard CJ, Asselin BL, Harris JP. Right atrial thrombi in children with cancer and indwelling catheters. J Pediatr 1996;128:841-6.

11. Gilon D, Schechter D, Rein AJ, et al. Right atrial thrombi are related to indwelling central venous catheter position: insights into time course and possible mechanism of formation. Am Heart J 1998;135:457-62.

12. P. T. Murphy, M. Sivakumaran, K. Ghosh, C. S. Chapman, and J. K. Wood, "Non-fatal massive right atrial thrombus associated with Hickman catheter in an adult receiving chemotherapy for acute leukaemia," Postgraduate Medical Journal 1994, vol. 70, no. 825, pp. 520-521

13. Kroger K, Grutter R, Rudofsky G, Fink H, Niebel W. Followup after Port-a-Cath-induced thrombosis. J Clin Oncol 2002;20:2605-6.

14. Chien-Yu Chen, Chih-Chung Liu, Wei-Zen Sun. EvidenceBased Review on Catheter-Related Thrombosis of the Implantable Venous Access Device. Tzu Chi Medical Journal, 2007;19(4):207-2 